\title{
An Oral Adsorbent, AST-120 Protects Against the Progression of Oxidative Stress by Reducing the Accumulation of Indoxyl Sulfate in the Systemic Circulation in Renal Failure
}

\author{
Kazuki Shimoishi, ${ }^{1,4}$ Makoto Anraku, ${ }^{1}$ Kenichiro Kitamura, ${ }^{2}$ Yuka Tasaki, ${ }^{1}$ Kazuaki Taguchi, ${ }^{1}$ \\ Mitsuru Hashimoto, ${ }^{3}$ Eiko Fukunaga, ${ }^{4}$ Toru Maruyama, ${ }^{1}$ and Masaki Otagiri ${ }^{1,5}$
}

\begin{abstract}
Received October 11, 2006; accepted January 19, 2007
Purpose. The effect of AST-120, an oral adsorbent, on oxidative stress in the systemic circulation in chronic renal failure (CRF) was examined and the potential role of indoxyl sulfate (IS), an uremic toxin adsorbed by AST-120, in inducing the formation of reactive oxygen species (ROS) in the vascular system was studied, in vitro and in vivo.

Materials and methods. The level of oxidized albumin, a marker for oxidative stress in the systemic circulation was determined by HPLC, as previously reported. The mRNA levels of TGF- $\beta_{1}$ and Oat1 were measured by quantitative RT-PCR. The IS induced ROS generation in cultured human umbilical vein endothelial cells (HUVECs) was estimated using a fluorescence microplate reader.

Results. An increase in the ratio of oxidized to unoxidized albumin was determined using $5 / 6$ nephrectomized rats (CRF rats) compared to a control group. The ratio was significantly reduced in the group that received AST-120 of 4 weeks, suggesting that AST-120 inhibits oxidative stress in CRF. An anti-oxidative effect of AST-120 was also observed in CRF rats with a similar renal function. The ratio of oxidized albumin was correlated with serum IS levels in vivo. The same relationship was also observed in CRF rats with the continued administration of IS. In addition, IS dramatically increased the generation of ROS in both a dose- and time- dependent manner in HUVEC, suggesting that accumulated IS may play an important role in enhancing intravascular oxidative stress.

Conclusion. We propose that AST-120 reduces IS concentrations in the blood that induces ROS production in endothelial cells, thereby inhibiting the subsequent occurrence of oxidative stress in the systemic circulation in renal failure.
\end{abstract}

KEY WORDS: albumin oxidation; AST-120; chronic renal failure; indoxyl sulfate.

\section{INTRODUCTION}

Oxidative stress, which involves the production of excessive levels of reactive oxygen spices (ROS) is closely

\footnotetext{
${ }^{1}$ Department of Biopharmaceutics, Graduate School of Pharmaceutical Sciences, Kumamoto University, 5-1 Oe-honmachi, Kumamoto 862-0973, Japan.

${ }^{2}$ Department of Nephrology, Graduate School of Medical Sciences, Kumamoto University, Kumamoto 860-8556, Japan.

${ }^{3}$ Department of Pathopharmacology, Graduate School of Pharmaceutical Sciences, Kumamoto University, Kumamoto 862-0973, Japan.

${ }^{4}$ Department of Pharmacy, Japanese Red Cross, Kumamoto Hospital, Kumamoto 861-8520, Japan.

${ }^{5}$ To whom correspondence should be addressed. (e-mail: otagirim@ gpo.kumamoto-u.ac.jp)

ABBREVIATIONS: CRF, chronic renal failure; CVD, cardiovascular disease; HPLC, high-performance liquid chromatography; HSA, human serum albumin; HUVEC, human umbilical vein endothelial cell; IS, indoxyl sulfate; OAT, organic anion transporter; ROS, reactive oxygen spiecies; TGF- $\beta_{1}$, transforming growth factor- $\beta_{1}$; TIMP-1, tissue inhibitor of metalloproteinease.
}

related to the progression of renal failure (1). Furthermore, the management of cardiovascular disease (CVD) is an important issue in cases of chronic renal failure (CRF) patients, and oxidative stress has been speculated to greatly contribute to such onset $(2,3)$. However, the development of effective treatment methods for reducing oxidative stress has also been sought on behalf of CRF.

The oral carbonaceous adsorbent, AST-120 (Kremezin $\left.{ }^{\circledR}\right)$, has been used in pre-dialysis, uremic stage renal failure patients to adsorb biologically active substances, the so-called uremic toxins, in the circulation that accumulate during CRF, thereby prolonging the progression of $\mathrm{CRF}$ and the interval to the inception of dialysis (4-7). In nephrectomy animal models, the administration of AST-120 attenuates the progression of renal failure without affecting the plasma rennin-angiotensin system or protein intake and reduces the gene expression of transforming growth factor- $\beta_{1}$ (TGF- $\beta_{1}$ ), a tissue inhibitor of metalloproteinease (TIMP-1) and pro- $\alpha 1$ (I) collagen in the kidney (8-10). However, details of the mechanism of pharmacological action of AST-120 remain unclear.

Indoxyl sulfate (IS), a naturally occurring metabolite of tryptophan, is considered to be a key target uremic toxin for AST-120 (11). The toxin is thought to be involved in the 
progression of CRF because it is present in high levels in the serum of patients with $\mathrm{CRF}$ and causes tubulointerstitial injuries in rodent models $(12,13)$. Several researchers have recently reported that IS has the potential to produce oxidative stress in renal proximal tubular cells and mesangial cells $(10,14)$. Moreover, supplementation of excessive dietary tryptophan, a precursor of IS, was reported to enhance plasma lipid peroxidation in rats (15). Based on these observations, we hypothesize that such oxidative stresses by IS can occur, not only at the tissue level in situ, but also in the vascular system, and if so, AST-120 could be useful for reducing oxidative stress in CRF. However, it is not clear whether AST-120 is associated with the reduction in oxidative stress.

In this study, we examined the effect of AST-120 on oxidative stress in CRF rats, to elucidate the potential role for AST-120 as an antioxidant in the systemic circulation. Oxidative stress was evaluated by monitoring oxidized serum albumin levels, a sensitive marker for protein oxidation in the systemic circulation (16). Furthermore, we investigated the effect of IS on ROS production in endothelial cells to verify the mechanism of the antioxidant activity of AST-120 in CRF.

\section{MATERIALS AND METHODS}

\section{Materials}

AST-120 was a generous gift from KUREHA CORPORATION (Tokyo, Japan). Indoxyl sulfate was purchased from Sigma Chemical (St. Louis, MO, USA). 5-(and-6)-chloromethyl-2', 7'dichlorodihydrofluorescein diacetate (CM-H $\left.{ }_{2} \mathrm{DCFDA}\right)$ was purchased from Molecular Probes (Leiden, Netherlands). HUVECs were purchased from Dainippon Sumitomo Pharma Co., Ltd (Osaka, Japan). All chemicals were of analytical grade.

\section{Experimental Design}

Male Wister rats were purchased from Seac Yoshitomi, Ltd. (Fukuoka, Japan). Five-week-old male rats weighing 140-150 g were subjected to a 5/6 nephrectomy as previously described $(17,18) .6$ weeks after the operation, the treated rats showed increased serum concentrations of creatinine (0.9 approximately $1.0 \mathrm{mg} / \mathrm{dl})$, blood urea nitrogen (BUN) (80 approximately $90 \mathrm{mg} / \mathrm{dl}$ ), and reduced levels of creatinine clearance (1.12 approximately $1.24 \mathrm{ml} / \mathrm{min})$. Age-matched Sham-operated rats were used for comparison $(n=7)$. These rats were divided into three groups as follows: (a) untreated nephrectomized group (untreated CRF rats) $(n=7)$. (b) AST-120 treated nephrectomized group (AST-120 treated CRF rats). These rats received standard rat chow and $4.5 \mathrm{~g} / \mathrm{kg}$ body wt of AST-120 for 10 weeks $(n=7)$. (c) Sham-operated rats $(n=7)$. Untreated CRF rats were pair-fed with the same amount of chow as the AST-120 treated CRF rats, and the normal rats were fed with standard solid chow ad-libitum. AST-120 was administered with the chow. After the administration of AST-120 for 10 weeks, all rats were sacrificed and their tissues were obtained.

\section{Biochemical Studies}

Serum creatinine (S-Cr), blood urea nitrogen (BUN), creatinine clearance $(\mathrm{Ccr})$, urinary protein, and serum IS were measured according to methods described previously $(5,8,9)$.

\section{RNA Isolation and Quantitative RT-PCR Analysis}

Total RNA was extracted from the frozen kidneys with RNAzol B (TEL-TEST, Friendswood, TX, U.S.A.) according to the manufacturer's instructions. The purity and quantity of the RNA preparation were determined by measuring the optical densities at 260 and $280 \mathrm{~nm}$. Quantitative two-step reverse-transcriptase (RT)-PCR using the LightCycler system (Roche Diagnostics) was performed to estimate the mRNA levels of TGF- $\beta_{1}$ and organic anion transporter 1 (Oat 1). The LightCycler system consists of a rapid PCR cycler and fluorescence-detection component, which allows real-time monitoring of fluorescence during PCR amplification (19). SYBR Green I (Roche Diagnostics), a double-standard DNA-binding fluorescent dye, was chosen to monitor cDNA amplification. The procedure of RT-PCR and quantification was described previously (9). The quantitative evaluation of mRNA levels is expressed as the ratio of that to GAPDH mRNA.

\section{Chromatographic Analysis of Serum Albumin in Rats}

HPLC was performed as described by Hayashi et al. (20). Sera samples obtained from each rat were immediately frozen and stored at $-80^{\circ} \mathrm{C}$ until used for HPLC analysis. HPLC analysis of $10 \mu \mathrm{l}$ aliquots of each serum was performed using a Shodex Asahipak ES-502N column (Showa Denko Co., Ltd., Tokyo, Japan; column temperature; $35^{\circ} \mathrm{C} \pm 0.5^{\circ} \mathrm{C}$ ). The HPLC system consisted of an L-6200 intelligent pump equipped with a gradient programmer and an F-1050 fluorescence detector (JASCO International Co., Ltd., Tokyo, Japan). Elution was performed using a linear gradient with increasing concentrations of ethanol from 0 to $10 \%$ with the serum dissolved in a mixuture of $0.05 \mathrm{~mol} / \mathrm{l}$ sodium acetate and $0.40 \mathrm{~mol} / \mathrm{l}$ sodium sulfate $(\mathrm{pH} 4.85)$ at a flow rate of $1.0 \mathrm{ml} / \mathrm{min}$. From the HPLC profiles of rat serum albumin, the ratios of oxidized to unoxidized albumin were estimated by dividing the area of the reduced form (rat mercaptalbumin (RMA)) to the oxidized form (rat non-mercaptalbumin (RNA)). The level of RNA/RMA ratio was estimated by the redox states of 34-Cys of rat serum albumin because Cys is highly accessible to reactive oxygen species. Therefore, the level of RNA/RMA ratio represents an appropriate marker of oxidative stress in CRF (20).

\section{Chromatographic Analysis of IS in Rats}

A $50 \mu \mathrm{l}$ aliquot of serum was added directly to $100 \mu \mathrm{l}$ of acetonitrile. After centrifugation at 3,000 g for $10 \mathrm{~min}$, the supernatant was assayed by HPLC. The HPLC system consisted of an L-6200 intelligent pump (Hitachi, Tokyo, Japan) and either an F-1050 fluorescence spectrophotometer (Hitachi). A column of LiChrosorb RP-18 (Cica Merck, 
Table I. Biochemical Data of Rats

\begin{tabular}{|c|c|c|c|c|c|c|}
\hline & \multicolumn{2}{|c|}{ Sham-Operated rats } & \multicolumn{2}{|c|}{ CRF Rats } & \multicolumn{2}{|c|}{ AST-120 Treated CRF Rats } \\
\hline & Week 0 & Week 10 & Week 0 & Week 10 & Week 0 & Week 10 \\
\hline BUN (mg/dl) & $20.0 \pm 3.0$ & $16.0 \pm 6.0$ & $80 \pm 15^{*}$ & $101 \pm 14^{*}$ & $90 \pm 15^{*}$ & $92 \pm 17^{*}$ \\
\hline Urinary protein $(\mathrm{mg} /$ day $)$ & $25.9 \pm 7.7$ & $30.3 \pm 8.2$ & $80.4 \pm 13^{*}$ & $160.4 \pm 22^{*}$ & $82.8 \pm 14.4^{*}$ & $110.8 \pm 18.3^{* \dagger}$ \\
\hline Serum IS $(\mu \mathrm{M})$ & $0.80 \pm 0.08$ & $1.20 \pm 0.11$ & $5.63 \pm 1.95^{*}$ & $17.4 \pm 0.80^{*}$ & $6.61 \pm 0.74^{*}$ & $6.67 \pm 2.75^{* \dagger}$ \\
\hline Serum Cr (mg/dl) & $0.39 \pm 0.14$ & $0.38 \pm 0.14$ & $0.93 \pm 0.16^{*}$ & $1.96 \pm 0.15^{*}$ & $1.01 \pm 0.17^{*}$ & $1.28 \pm 0.42^{* \dagger}$ \\
\hline $\operatorname{Ccr}(\mathrm{ml} / \mathrm{min})$ & $2.5 \pm 0.31$ & $2.4 \pm 0.18$ & $1.12 \pm 0.27^{*}$ & $0.72 \pm 0.21^{*}$ & $1.24 \pm 0.23^{*}$ & $1.28 \pm 0.22^{* \dagger}$ \\
\hline
\end{tabular}

Data are means $\pm \mathrm{SE} .{ }^{*} P<0.05$ versus sham-operated rats. ${ }^{\dagger} P<0.05$ versus $\mathrm{CRF}$ rats

Tokyo, Japan) was used as a stationary phase. The mobile phase consisted of acetate buffer (0.2 M, pH 4.5)/acetonitrile. The flow rate was $1.0 \mathrm{ml} / \mathrm{min}$. IS was detected by means of a fluorescence monitor. The excitation/emission wavelengths were $300 / 400 \mathrm{~nm}(21)$.

\section{IS Administration to CRF Rats}

Two weeks after the 5/6-nephrectomy, ten rats were divided into pairs such that both rats in each pair exhibited almost the same levels of S-Cr and urine protein, and five rats were then intraperitoneary administered IS daily at a dose of $50 \mathrm{mg} / \mathrm{kg}$ body wt.

\section{Cell Culture and the Measurements of ROS in HUVECs}

Among endothelial cells, HUVECs are frequently used to study the mechanism of alternation of endothelial cell function induced by oxidative stress (22). Therefore, we used HUVECs in the present study as a model of human endothelial cells. HUVECs were cultured as previously described (23), and used in experiments at passages between 3 and 5. To investigate the effect of IS on the generation of ROS in HUVECs, we measured the fluorescence intensities of 5-(and-6)-chloromethyl-2', 7'-dichlorodihydrofluorescein diacetate (CM$\mathrm{H}_{2}$ DCFDA) as a ROS probe. HUVECs $\left(10^{4}\right.$ cell/well $)$ were pre-incubated in 96-well plates for $24 \mathrm{~h}$ at $37^{\circ} \mathrm{C}$ in culture medium. Confluent HUVECs were then incubated with $10 \mu \mathrm{M}$ $\mathrm{CM}-\mathrm{H}_{2} \mathrm{DCFDA}$ for $30 \mathrm{~min}$ at $37^{\circ} \mathrm{C}$ in serum-free medium. After the removal of the media from wells, IS at $37^{\circ} \mathrm{C}$ in serumfree medium with or without $4 \%$ HSA was added to the cells. The fluorescence intensity was measured at $490 \mathrm{~nm}$ excitation and $530 \mathrm{~nm}$ emission using a fluorescence microplate reader (CORONA Multi Microplate Reader). The mean fluorescence intensity ratio (MFI ratio) is presented as the percentage of the control value, after subtraction of background fluorescence.

AST-120 treated $0.5 \mathrm{mM}$ IS was prepared as follows. A stock solution of $0.5 \mathrm{mM}$ IS was co-incubated with $1 \mathrm{~g} / \mathrm{dl} \mathrm{AST-120}$ for $3 \mathrm{~h}$ at $37^{\circ} \mathrm{C}$ and the supernatants were filtered by ultrafiltration.

\section{Statistics}

Statistical significance was evaluated using the two-tailed, unpaired Student's $t$ test for comparisons between two means, or ANOVA analysis followed by Newman-Keuls method for more than two means. Results are reported as mean $\pm \mathrm{SE}$.

\section{RESULTS}

\section{Biochemical Parameters in Vivo}

Prior to examining the effect of AST-120 on oxidative stress in CRF, the biochemical parameters related to the progression of renal failure in sham rats (control), CRF rats and CRF rats to be treated with AST-120 were obtained (Table I). At the end of week 10, significant increases in serum creatinine, BUN, urinary protein and serum IS levels with a significant decrease in creatinine clearance were observed in CRF rats, when compared with sham rats. In contrast, AST120 treated CRF rats showed decreases in serum creatinine, urinary protein and serum IS, with a significant increase in creatinine clearance than the untreated CRF rats. These results are consistent with previous reports $(5,8,9)$.

Figure 1 shows the renal expression of TGF- $\beta_{1}$ and Oat 1 of each rat group at the end of the treatment. As previously reported, TGF- $\beta_{1}$ was significantly up-regulated in the CRF rats in comparison with the sham rats, but it was decreased upon AST-120 treatment (Fig. 1a) (9). In contrast, renal expression of Oat1 was significantly decreased in CRF rats. This decrease in gene expression was significantly retrieved by AST-120 treatment (Fig. 1b). These phenomena were also in agreement with the previous reports $(9,24)$.

\section{Effects of AST-120 on Oxidative Stress}

Figure 2 shows the ratio of oxidized albumin as an index of oxidative stress in the systemic circulation in each group. The values of the ratio in untreated CRF rats were
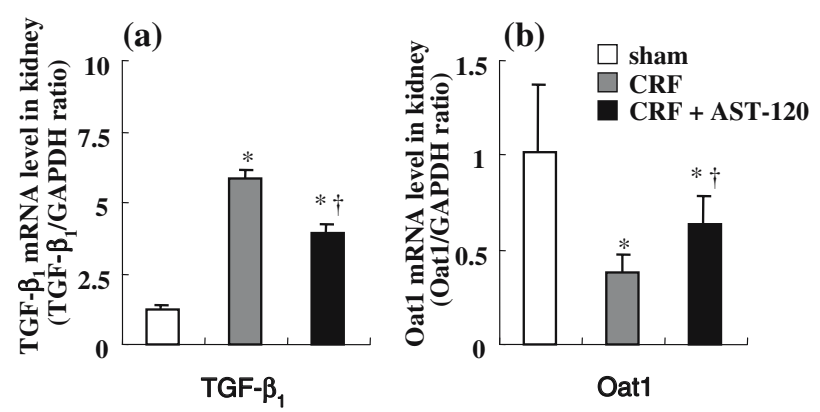

Fig. 1. Quantitative RT-PCR analysis of gene expression for TGF- $\beta_{1}$ and Oat1. Results are expressed as the mean \pm SE. $(n=3-6)$ ${ }^{*} P<0.05$ compared to sham-operated rats; $\dagger P<0.05$ compared to untreated CRF rats. 


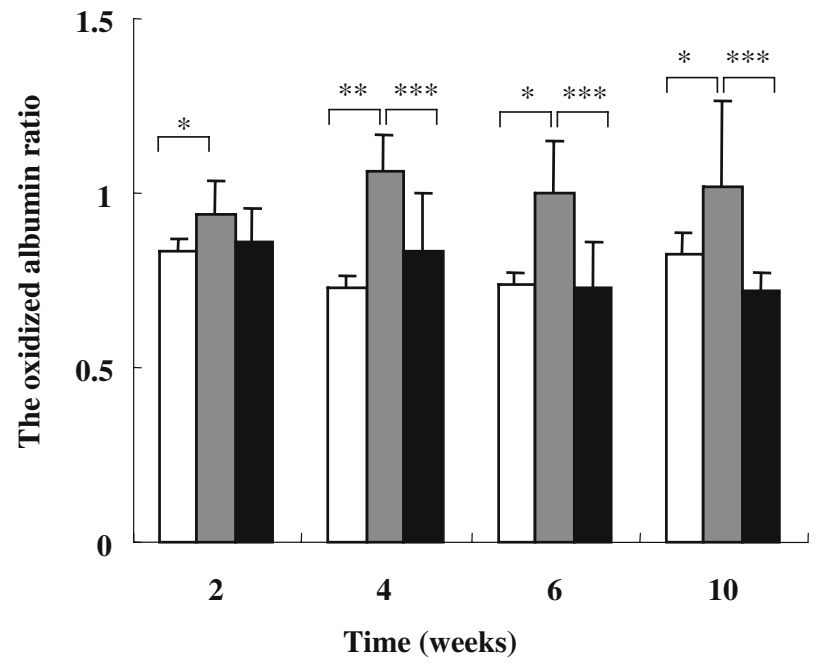

Fig. 2. Levels of the ratio of oxidized to unoxidized albumin from AST-120 treated CRF rats $(\square)$, untreated CRF rats $(\square)$ and shamoperated rats $(\square)$. $* P<0.05, * * P<0.01$ compared with shamoperated rats. $* * * P<0.05$ compared with untreated $\mathrm{CRF}$ rats. Results are expressed as the mean \pm SE. $(n=3-6)$.

significantly higher than in control group. Interestingly, a significant reduction in the oxidized albumin ratio was observed after 4 weeks of AST-120 administration $(p<0.05)$, suggesting that AST-120 reduces oxidative stress in CRF rats. In order to determine whether this anti-oxidant potency of AST-120 was due to the adsorption of uremic toxins or to inhibiting the progression of CRF, we examined the effect of AST-120 treatment on the oxidized albumin ratio in CRF rats with a similar renal function $(0.75<\mathrm{S}-\mathrm{Cr}<1.25)$. As shown in Fig. 3, the administration of AST-120 also significantly decreased the ratio of oxidized albumin in CRF rats even though they had similar renal functions.

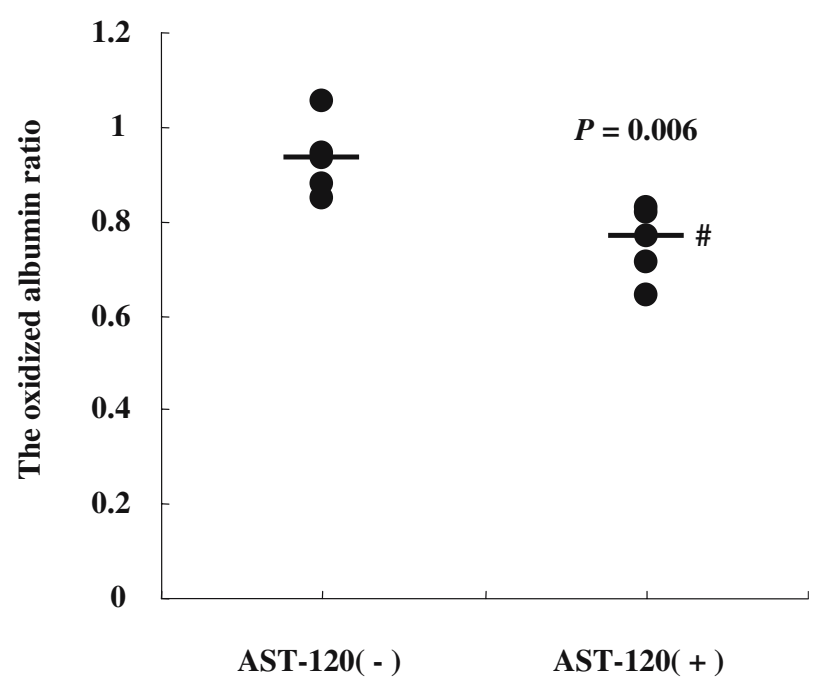

Fig. 3. Levels of the ratio of oxidized to unoxidized albumin from AST-120 treated CRF rats and untreated CRF rats with the same renal function $(0.75<\mathrm{S}-\mathrm{Cr}<1.25)$. Horizontal bars represent the median oxidized albumin ratio for each group. \# $P=0.006$ compared with untreated CRF rats $(n=5)$.
Relationship between Oxidative Stress and Serum IS Levels

To examine the factors that influence oxidative stress in CRF rats, serum IS concentrations and the extent of albumin oxidation were measured in control and CRF rats with or without AST-120 treatment. Figure 4 shows the relationship between serum IS concentration and the ratio of oxidized albumin. The values of the ratio were closely correlated with serum IS levels $(\mathrm{R}=0.628, P<0.01)$, indicating that the accumulation of IS is likely to induce oxidative stress in CRF rats. However, the administration of AST-120 dramatically reduced the oxidized albumin ratio nearly to the level of the control. This can be attributed to the lowering of accumulated IS levels by AST-120.

\section{Effects of IS on Oxidative Stress}

To confirm the possibility that IS induces oxidative stress in CRF rats, we further examined the effects of exogenously administrated IS on the oxidized albumin ratio in CRF rats. IS was intraperitoneary administered daily at a dose of $50 \mathrm{mg} /$ $\mathrm{kg}$ body wt. As shown in Fig. 5, there was a relationship between the increases in IS concentration and the ratio of oxidized albumin. These findings further demonstrate the production of oxidative stress by IS accumulation in CRF.

\section{Effects of IS on ROS Production in HUVECs}

To clarify whether the oxidative stress induced by IS occurs in the vascular system, we investigated the effect of IS on ROS generation in HUVECs. As shown in Fig. 6a, IS increased ROS production in HUVECs in a time-dependent

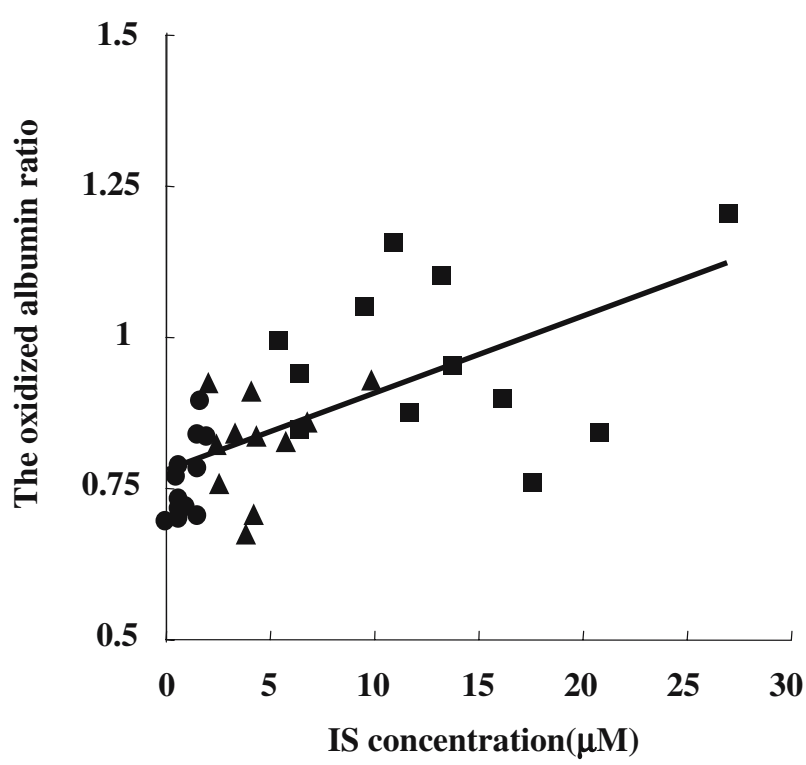

Fig. 4. Relationship between serum IS concentration and the levels of oxidized albumin. Serum IS concentration and the ratio of oxidized to unoxidized albumin from sham-operated rats (closed circle) and CRF rats with (closed triangle) or without (closed square) AST-120 are plotted, and the line shows a linear regression of IS concentration and the levels of oxidized albumin $(n=36, \mathrm{R}=0.628$, $P<0.01)$. 


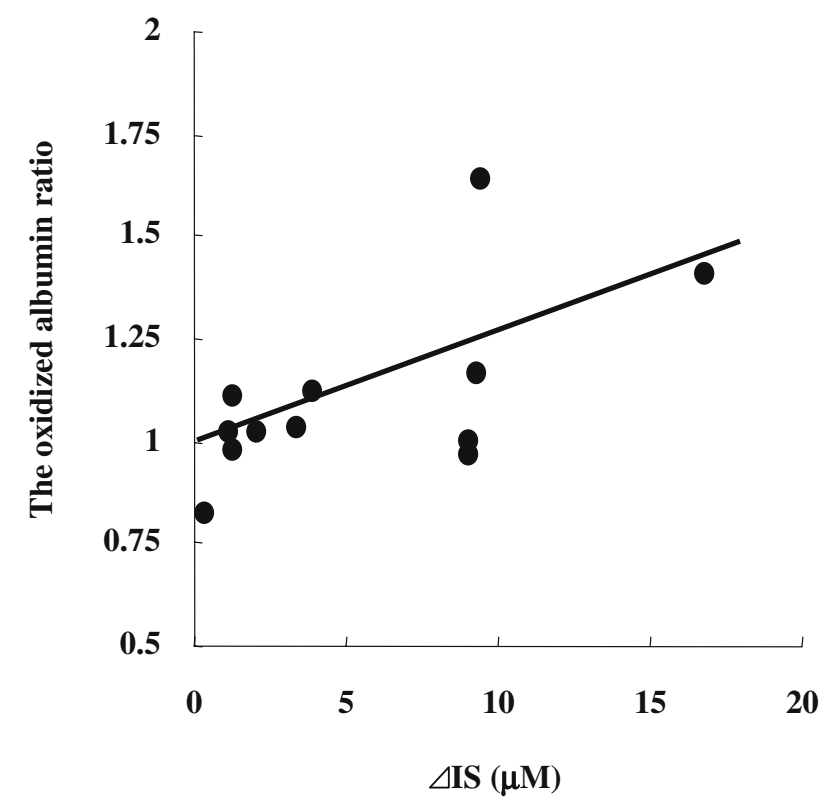

Fig. 5. Relationship between the increase in serum IS concentration and the ratio of oxidized to unoxidized albumin in IS treated and untreated rats. IS was administered daily at a dose of $50 \mathrm{mg} / \mathrm{kg}$ body wt to CRF rats. The increase in IS concentration ( $\Delta$ IS) and the levels of oxidized albumin are plotted, and the line shows linear regression of $\Delta \mathrm{IS}$ and the levels of oxidized albumin $(n=10, \mathrm{R}=0.621, \mathrm{P}<0.05)$.

manner and the increase reached a plateau after $3 \mathrm{~h}$. Therefore, following experiments were performed for a $3 \mathrm{~h}$ incubation. It was found that the ROS productions in HUVECs were significantly dependent on the amount of IS added (Fig. 6b). However, such increase in ROS production by IS $(0.5 \mathrm{mM})$ was decreased to the control level by AST120 pretreatment. Because IS is mainly bound to albumin in blood, we also evaluated the effect of IS in the presence of $4 \%$ HSA in the medium. Although to the lesser extent, IS at $0.5 \mathrm{mM}$ significantly increased the ROS generation in HUVECs with 4\% HSA (Fig. 6c). In 4\% HSA medium, IS is highly bound to albumin (>90\%) (25), and then free IS may not sufficiently explain this phenomenon. Very recently, similar findings have been found for the enhanced proliferation of rat vascular smooth muscle cells by IS in the presence of albumin (26). These results suggest that IS may directly stimulate ROS generation through endotherial cells, leading to the development of oxidative stress in the systemic circulation.

\section{DISCUSSION}

Oxidative stress is a pathogenic element of great importance in uremic patients, and has a great impact on their survival. Thus, the development of effective anti-oxidant therapy is warranted in CRF. One proposed mechanism of oxidative stress in CRF is the accelerated production of oxidants, such as uremic toxins and their reduced renal clearance. Thus, the removal of such substances from the systemic circulation may lead to a reduction in oxidative stress in CRF. AST-120, a molecular adsorbent, was developed to remove toxic substances from the bodies of uremic patients. The purpose of this study was to identify the potential role for AST-120 as a systemic anti-oxidant in CRF in vitro and in vivo. We monitored the ratio of oxidized to unoxidized albumin as a marker for oxidative stress in the systemic circulation due to its simplicity, rapidity and sensitivity for evaluating oxidative stress in vivo because the oxidation of albumin accounts for almost all of the excess plasma protein oxidation in uremic patients. In fact, we previously demonstrated that intravenous iron induced oxidative stress in hemodialysis patients by this technique (16).

One result of great interest was that the oxidative stress observed in CRF rats was significantly inhibited in an AST120 administration group (Figs. 2 and 3), suggesting the possibility that AST-120 inhibits oxidative stress in the serum of CRF rats. Reports to date have only focused on the localized effects of AST-120 in the kidney, such as inhibition of the genetic expression of TGF- $\beta_{1}$, TIMP-1, and pro$\alpha 1$ collagen (13) and suppression of the level of urine oxidant, 8-OHdG (27), but the antioxidant effect of AST-120 on the systemic circulation is, to our knowledge, the first such finding.

Since AST-120 is not absorbed, it is unlikely that the mechanism of AST-120 antioxidant activity directly participates in radical scavenging in blood; rather, we hypothesize an indirect manifestation of activity, in which substances causing oxidative stress or their precursors are adsorbed in the gastrointestinal tract, thus suppressing their levels in blood. This was supported by a correlation between the serum concentration of IS, a primary substance adsorbed by AST-120, and the oxidized albumin ratio in CRF rats. Nonetheless, it would be premature to reach a conclusion from these data alone as to whether an elevation in albumin oxidation results from the progression of renal failure or from the accumulation of IS. To clarify this issue, we performed a continued administration of IS to CRF rats to
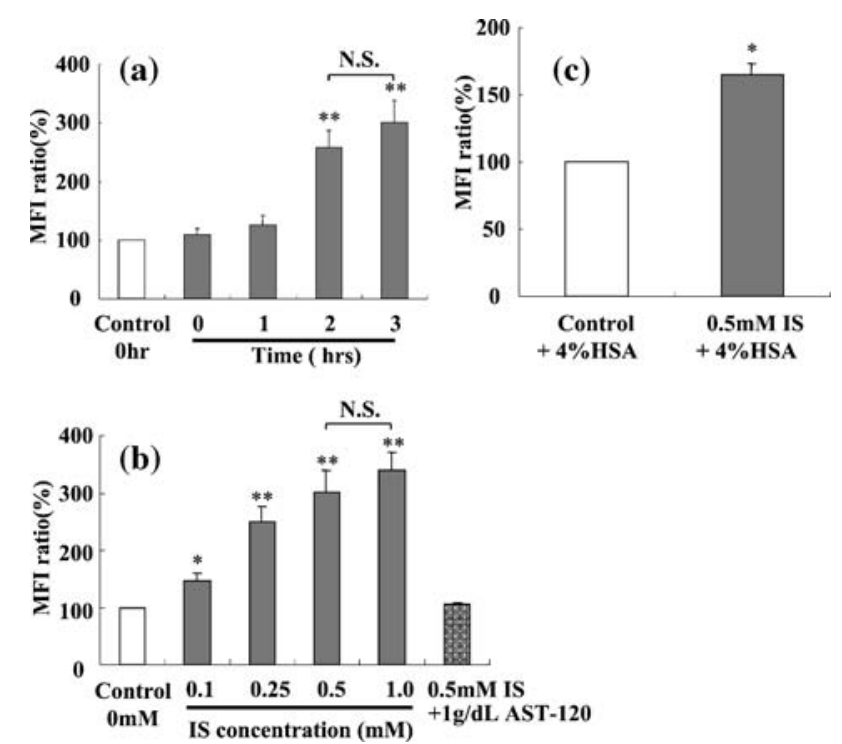

Fig. 6. Effects of IS on ROS production of HUVECs. HUVEC $\left(10^{4}\right.$ cell/well) in 96-well plates were pre-incubated with $10 \mu \mathrm{M} \mathrm{CM}$ $\mathrm{H}_{2}$ DCFDA for $30 \mathrm{~min}$ at $37^{\circ} \mathrm{C}$ in serum-free medium. a: cells were added with $0.5 \mathrm{mM}$ IS for $0-3 \mathrm{~h}$ at $37^{\circ} \mathrm{C}$. b: cells were added with indicated amount of IS and AST-120 treated $0.5 \mathrm{mM}$ IS for $3 \mathrm{~h}$ at $37^{\circ} \mathrm{C}$. c: cells were added with $0.5 \mathrm{mM}$ IS and $4 \%$ HSA for $3 \mathrm{~h}$ at $37^{\circ} \mathrm{C}$, and the mean fluorescence intensities were measured. Values are mean $\pm \operatorname{SE}(n=4) . * P<0.05, * * P<0.01$ compared with control. 
investigate how variations in IS serum levels affected albumin oxidation. As anticipated, albumin oxidation increased with increasing levels of IS. These results suggest that the accumulation of IS in the blood may play an important role in the elevation of oxidative stress in the systemic circulation in CRF.

Previous studies of the induction of ROS by IS were limited to the use of renal tubule cells (OK cells) and mesanginal cells, but no findings in intravascular cell lines have been reported $(10,14)$. However, our results clearly show that IS also induce ROS production in HUVECs to a remarkably extent and this was largely inhibited by AST-120 pretreatment. Such ROS production by IS was dose- and time-dependent, as has also been reported in OK cells (28). Given that the accumulation of IS in the blood can reach $1 \mathrm{mM}$ in CRF, it is quite possible that IS induces oxidative stress intravascularly in CRF patients. The mechanism by which IS induces ROS production in HUVECs is not yet known from the present limited results. We previously reported that IS was taken up by proximal tubular cells by organic anion transporters (Oat1, Oat3) and Motojima et al. demonstrated that such cellular uptake causes proximal tubular injury $(28,29)$. In addition, Gelasco et al. found that IS stimulates the production of intracellular and extracellular ROS in rat mesanginal cells, through a pathway that most likely involves NADPH oxidase or NADPH like oxidase (14). However, determining the molecular mechanism by which IS induces the ROS production in the endothelial cells will require further in vitro studies, which are now underway in our laboratory. Based on those findings, we proposed a mechanism by which oxidized albumin was produced by IS induced endothelial cell damage. This dose not exclude the possibility that the accumulation of oxidized albumin is thought to be generated by IS within the kidney, such as proximal tubules or mesangium cells. In addition, the states of oxidized albumin in kidney have been believed the end products via oxidation. However, the oxidized albumin that we focused on blood is the early product because the oxidative state of albumin is estimated from the redox state of 34Cys which is sensitive to the early state of oxidation process. In any case, AST-120 decreased the oxidative stress in systemic circulation via inhibiting of accumulated IS in kidney as well as blood.

To date, AST-120 has been used in pre-dialysis and uremic-stage renal failure patients $(30,31)$. However, the present results suggest a new potential use for AST-120 as an antioxidant in renal failure. We therefore propose that, from the perspective of antioxidant therapy, the initiation of AST120 administration is preferable at a stage earlier than the conventional state of pre-dialysis uremia. Otherwise, we feel it may be necessary to administer AST-120 to hemodialysis patients as an antioxidant because plasma levels of IS undergo a significant increase during each hemodialysis session.

In the current studies, we demonstrated the antioxidative potential of AST-120 in the systemic circulation in CRF rats. From these results, we hypothesized that AST-120 reduces IS concentrations in the blood that induces ROS production in endothelial cells, thereby inhibiting the subsequent occurrence of oxidative stress in the systemic circulation in CRF. Thus, the antioxidative effect of AST-120 is unique and differs from that of typical, conventional anti- oxidants such as renin-angiotensin inhibitors, antioxidant vitamins and $\mathrm{N}$-acetyl cysteine. AST-120 can therefore be co-administered with such agents, portending a new strategy for antioxidative treatment in cases of renal failure.

\section{ACKNOWLEDGEMENTS}

We wish to thank the KUREHA CORPORATION (Tokyo, Japan) for the generous gift of AST-120.

\section{REFERENCES}

1. J. Himmelfarb. Linking oxidative stress and inflammation in kidney disease: which is the chicken and which is the egg? Sem. Dial. 17:449-454 (2004).

2. U. Singh and I. Jialal. Oxidative stress and atherosclerosis. Pathophysiology 13:129-142 (2006).

3. J. Fort. Chronic renal failure: a cardiovascular risk factor. Kidney Inter. Suppl. 99:S25-S29 (2005).

4. Y. Yoshida, T. Sakai, and M. Ise. Effects of oral adsorbent in the rat model of chronic renal failure. Nephron 62:305-314 (1992).

5. I. Aoyama, T. Miyazaki, and T. Niwa. Preventive effects of an oral sorbent on nephropathy in rats. Miner. Electrolyte Metab. 25:365-372 (1999).

6. A. Owada, M. Nakao, J. Koike, K. Ujiie, K. Tomita, and T. Shiigai. Effects of oral adsorbent AST-120 on the progression of chronic renal failure: a randomized controlled study. Kidney Inter. Suppl. 63:S188-S190 (1997).

7. T. Sanaka, N. Sugino, S. Teraoka, and K. Ota. Therapeutic effects of oral sorbent in undialyzed uremia. Am. J. Kidney Dis. 12:97-103 (1988).

8. T. Miyazaki, I. Aoyama, M. Ise, H. Seo, and T. Niwa. An oral sorbent reduces overload of indoxyl sulphate and gene expression of TGF-beta1 in uraemic rat kidneys. Nephrol. Dial. Transplant. 15:1773-1781 (2000).

9. I. Aoyama, A. Enomoto, and T. Niwa. Effects of oral adsorbent on gene expression profile in uremic rat kidney: cDNA array analysis. Am. J. Kidney Dis. 41:S8-S14 (2003).

10. M. Motojima, A. Hosokawa, H. Yamato, T. Muraki, and T. Yoshioka. Uremic toxins of organic anions up-regulate PAI-1 expression by induction of NF-kappaB and free radical in proximal tubular cells. Kidney Int. 63:1671-1680 (2003).

11. T. Niwa, T. Miyazaki, N. Hashimoto, H. Hayashi, M. Ise, Y. Uehara, and K. Maeda. Suppressed serum and urine levels of indoxyl sulfate by oral sorbent in experimental uremic rats. Am. J. Nephrol. 12:201-206 (1992).

12. M. Motojima, F. Nishijima, M. Ikoma, T. Kawamura, T. Yoshioka, A. B. Fogo, T. Sakai, and I. Ichikawa. Role for "uremic toxin" in the progressive loss of intact nephrons in chronic renal failure. Kidney Int. 40:461-469 (1991).

13. T. Miyazaki, M. Ise, H. Seo, and T. Niwa. Indoxyl sulfate increases the gene expressions of TGF-beta 1, TIMP-1 and proalpha 1(I) collagen in uremic rat kidneys. Kidney Inter. Suppl. 62:S15-S22 (1997).

14. A. K. Gelasco and J. R. Raymond. Indoxyl sulfate induces complex redox alterations in mesangial cells. Am. J. Physiol. Renal Physiol. 290:F1551-F1558 (2006).

15. Y. Ohta, and D. Sahashi. L-tryptophan administration promotes the reversion of pre-established chronic liver injury in rats treated with carbon tetrachloride. J. Nutr. Biochem 13:550 (2002).

16. M. Anraku, K. Kitamura, A. Shinohara, M. Adachi, A. Suenga, T. Maruyama, K. Miyanaka, T. Miyoshi, N. Shiraishi, H. Nonoguchi, M. Otagiri, and K. Tomita. Intravenous iron administration induces oxidation of serum albumin in hemodialysis patients. Kidney Int. 66:841-848 (2004).

17. L. Ji, S. Masuda, H. Saito, and K. Inui. Down-regulation of rat organic cation transporter rOCT2 by 5/6 nephrectomy. Kidney Int. 62:514-524 (2002). 
18. A. Takeuchi, S. Masuda, H. Saito, T. Doi, and K. Inui. Role of kidney-specific organic anion transporters in the urinary excretion of methotrexate. Kidney Int. 60:1058-1068 (2001).

19. T. Aoshima, Y. Sekido, T. Miyazaki, M. Kajita, S. Mimura, K. Watanabe, K. Shimokata, and T. Niwa. Rapid detection of deletion mutations in inherited metabolic diseases by melting curve analysis with LightCycler. Clin. Chem. 46:119-122 (2000).

20. T. Hayashi, K. Suda, H. Imai, and S. Era. Simple and sensitive high-performance liquid chromatographic method for the investigation of dynamic changes in the redox state of rat serum albumin. J. Chromatogr. B Analyt. Technol. Biomed. Life Sci. 25:139-146 (2002).

21. Y. Tsutsumi, T. Deguchi, M. Takano, A. Takadate, W. E. Lindup, and M. Otagiri. Renal disposition of a furan dicarboxylic acid and other uremic toxins in the rat. J. Pharmacol. Exp. Ther. 303:880-887 (2002).

22. J. Jacobi, B. Kristal, J. Chezar, S. M. Shaul, and S. Sela. Exogenous superoxide mediates pro-oxidative, proinflammatory, and procoagulatory changes in primary endothelial cell cultures. Free Radic. Biol. Med. 39:1238-1248 (2005).

23. H. Li and U. Förstermann. Structure-activity relationship of staurosporine analogs in regulating expression of endothelial nitric-oxide synthase gene. Mol. Pharmacol. 57:427-435 (2000).

24. A. Enomoto, M. Takeda, A. Tojo, T. Sekine, SH. Cha, S. Khamdang, F. Takayama, I. Aoyama, S. Nakamura, H. Endou, and T. Niwa. Role of organic anion transporters in the tubular transport of indoxyl sulfate and the induction of its nephrotoxicity. J. Am. Soc. Nephrol.13:1711-1720 (2002).

25. T. Sakai, K. Yamasaki, T. Sako, U. Kragh-Hansen, A. Suenaga, and M. Otagiri. Interaction mechanism between indoxyl sulfate, a typical uremic toxin bound to site II, and ligands bound to site I of human serum albumin. Pharm. Res. 18:520-524 (2001).

26. H. Yamamoto, S. Tsuruoka, T. Ioka, H. Ando, C. Ito, T. Akimoto, A. Fujimura, Y. Asano, and E. Kusano. Indoxyl sulfate stimulates proliferation of rat vascular smooth muscle cells. Kidney Int. 69:1780-1785 (2006).

27. N. Nakagawa, N. Hasebe, K. Sumitomo, T. Fujino, J. Fukuzawa, T. Hirayama, and K. Kikuchi. An oral adsorbent, AST-120, suppresses oxidative stress in uremic rats. Am. J. Nephrol. 26:455-461 (2006).

28. M. Motojima, A. Hosokawa, H. Yamato, T. Muraki, and T. Yoshioka. Uraemic toxins induce proximal tubular injury via organic anion transporter 1-mediated uptake. Br. J. Pharmacol. 135:555-563 (2002)

29. T. Deguchi, H. Kusuhara, A. Takadate, H. Endou, M. Otagiri, and Y. Sugiyama. Characterization of uremic toxin transport by organic anion transporters in the kidney. Kidney Int. 65:162-174 (2004).

30. T. Niwa and M. Ise. Indoxyl sulfate, a circulating uremic toxin, stimulates the progression of glomerular sclerosis. J. Lab. Clin. Med. 124:96-104 (1994).

31. I. Aoyama and T. Niwa. An oral adsorbent ameliorates renal overload of indoxyl sulfate and progression of renal failure in diabetic rats. Am. J. Kidney Dis. 37:S7-S12 (2001). 